\title{
On the twin-prime problem, II
}

by

P. Tưrán (Budapest)

To the 100th anniversary of B. Riemann's death

1. The asymptotical determination of the number of twin primes not exceeding $x$ amounts essentially to the investigation of the sum

$$
S_{2}(x)=\sum_{n \leqslant x} \Lambda(n) \Lambda(n+2)
$$

or more generally for even $D$ to

$$
S_{D}(x)=\sum_{\substack{n \leq x \\(n, D)=1}} \Lambda(n) \Lambda(n+D) .
$$

Several interesting results "of approximative character" have been achieved since Brun; but the only method which could produce at least a plausible heuristical asymptotical formula for $S_{D}(x)$ itself was HardyLittlewood's circle method. Their formula (see [1], p. 42, conjecture B) asserted that for fixed even $D$ and $x \rightarrow \infty$ the relation

$$
S_{D}(x) \sim B_{1} x \prod_{\substack{p \mid D \\ p>2}} \frac{p-1}{p-2}
$$

holds with $\left({ }^{1}\right)$

$$
B_{1}=2 \prod_{p>2}\left(1-\frac{1}{(p-1)^{2}}\right) .
$$

(1) Or equivalently denoting by $\pi_{D}(x)$ the number of prime pairs $p_{1}, p_{2}$ with $p_{1}-p_{2}=D$ not exceeding $x$,

$$
\pi_{D}(x) \sim B_{1} \frac{x}{\log ^{2} x} \prod_{\substack{p \mid D \\ p>2}} \frac{p-1}{p-2} .
$$


Recently I observed that a much shorter heuristical deduction of (1.1) can be given which gives rise to new-type sieve methods. Starting namely from the formula

$$
\text { (1.3) } \quad \Lambda(m)=-\sum_{k \mid m} \mu(k) \log k \quad\left(=\sum_{k \mid m} \mu(k) \log \frac{x}{k} \text { for } m>1\right)
$$

we get at once

$$
\begin{aligned}
& S_{D}(x)=-\sum_{\substack{n \leq x \\
(n, D)=1}} \Lambda(n) \sum_{\substack{k \mid(n+D) \\
(k)}} \mu(k) \log k=-\sum_{\substack{k \leq x+D) \\
(k, D)=1}} \mu(k) \log k \sum_{\substack{n \leq x) \\
n=1 \\
(n, D)=1}} \Lambda(n) \\
& \sim-\sum_{\substack{(k, D)=1 \\
k \leqslant x+D}} \mu(k) \log k \sum_{\substack{n \leqslant x \\
n \equiv-D \bmod k}} \Lambda(n)
\end{aligned}
$$

Replacing the inner sum heuristically by $x / \varphi(k)$ one gets heuristically

$$
S_{D}(x) \sim x\left\{-\sum_{\substack{k \leq x+D \\(k, D)=1}} \frac{\mu(k) \log k}{\varphi(k)}\right\}
$$

But as the proof of Lemma III will show rigorously the sum in brackets

$$
\sim B_{1} \prod_{\substack{p \mid D \\ p>2}} \frac{p-1}{p-2}
$$

which gives the formula (1.1) of Hardy-Littlewood at once. In principle the argument could be remedied by using for $\sum_{n=x} \Lambda(n)$ instead of $x / \varphi(k)$

$$
\begin{gathered}
n=-D \ln \\
n=-D \bmod k
\end{gathered}
$$

an "exact" prime-formula of Riemann-type but this seems hopelessly complicated. Instead of doing so I realised in the first paper of this series (see [3]) that "sieving on the generating Dirichlet series" leads at once to the formula $(s=\sigma+i t, \sigma>1)$

$$
\begin{array}{r}
\sum_{\substack{n \leqslant N-2 \\
n \text { odd }}} \frac{\Lambda(n) \Lambda(n+2)}{n^{s}}+\sum_{\substack{4 \leqslant 2^{\lambda} \leqslant N-2 \\
(\lambda)}} \frac{\log 2 \cdot \Lambda\left(2^{\lambda-1}+1\right)}{2^{\lambda s}}+\sum_{n>N-2} \frac{b_{n}}{n^{s}} \\
=\sum_{\substack{k<N \\
k \text { odd }}} \frac{\mu(k) \log k}{\varphi(k)} \sum_{x \bmod k} \bar{\chi}(-2, k) \frac{L^{\prime}}{L}(s, k, \chi)
\end{array}
$$

and this offers several possibilities for a more elegant treatment and various conclusions. So I used the formula (1.4) to show that the infiniteness of the twin primes depends only upon "small" zeros of the Dirichlet $L(s, k, \chi)$-functions; it turned out on this way recently that among these zeros only those "near to the line $\sigma=\frac{1}{2}$ " have a significance for the problem. As I remarked in my paper [4], this "functiontheoretical sieve" method can be applied to a large class of "indefinite" problems of the additive prime number theory a typical of them being the twin prime problem. To formulate it in a general form (which is far from being most general) let the integers $x_{1}, \ldots, x_{l}$ be restricted to the sets $A_{1}, A_{2}, \ldots, A_{l}$ respectively and let $f\left(x_{1}, \ldots, x_{l}\right)=f(x)$ be any integer-valued function for which the number $h(v)$ of the solutions of the equation

$$
f(x)=\nu, \quad x_{j} \epsilon A_{j}, \quad j=1,2, \ldots, l,
$$

is finite, even the estimation $\left({ }^{2}\right)$

$$
h(v)<v^{B_{2}}
$$

holds. Let $h(v)$ be in addition such that for all positive integer $B$ 's the function-theoretical behaviour of the functions

$$
G_{B}(s, k, \chi) \stackrel{\text { def }}{=} \sum_{(v, B)=1} \frac{h(v) \chi(\nu, k)}{\nu^{s}}
$$

is at our disposal; this is the case e. g. when $h(v)$-apart perhaps from a numerical factor-is multiplicative, an example being $f=x_{1}^{2}+x_{2}^{2}$, the sets $A_{j}$ being identical to all integers. Let us consider with a positive integer $N>B$ the function

$$
\text { (1.8) } \quad K_{N}(s, B) \stackrel{\text { def }}{=} \sum_{\substack{k \leqslant N \\(k, B)=1}} \frac{\mu(k) \log (N / k)}{\varphi(k)} \sum_{\chi \bmod k)} \bar{\chi}(-B, k) G_{B}(s, k, \chi)
$$

(2) $e$ will stand throughout for positive constants (not necessarily the same) whose numerical values are irrelevant, $B_{1}, B_{2}, \ldots$ stand for positive constants whose whose numerical values are irrelevant, $B_{1}, B_{2}, \ldots$ stand for positive constants whose numerical values matter. The characters belonging to modulus $k$ will be denoted
(as in (1.4)) by $\chi(n, k)$, the corresponding $L$-function by $L(s, k, \chi)$, their non-trivial zeros by $\varrho=\varrho(k, \chi)=\beta+i \gamma \cdot p$ is always a prime, the $O$-and $o$-signs refer to $N \rightarrow \infty$. The empty sum means 0 , empty product 1 . $\tau(l)$ stands for the number of positive divisors of $l$. $\sum^{*}$ means that the summation is to be extended to primitive characters $\bmod k$ only. $\exp x$ stands for $e^{x}$. 
for $\sigma>B_{2}+1$. The series (1.7) can be inserted here and this gives

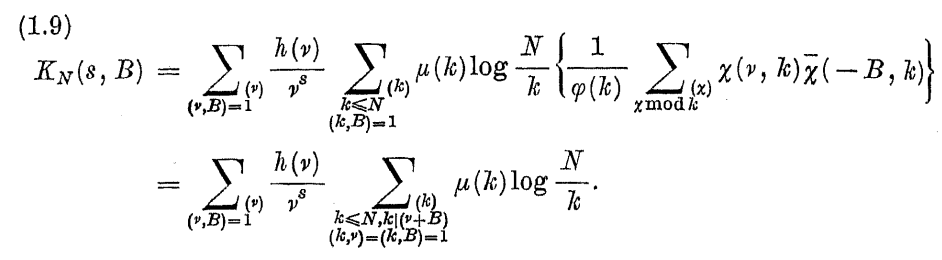

We may observe that the restrictions

$$
(k, v)=(k, B)=1
$$

are automatically satisfied; e.g. $p \mid z$ and $p \mid \nu$ would imply $p \mid B$, i.e. $(\nu, B)$ $>1$, which is excluded. Hence the inner sum is for $v \leqslant N-B$ owing to (1.3) equal to $A(\nu+B)$ and hence for $\sigma>B_{2}+1$

$$
K_{N}(s, B)=\sum_{\substack{\nu \leqslant N-B \\(\nu, B)=1}} \frac{h(v) \Lambda(\nu+B)}{\nu^{8}}+\sum_{\nu>N-B} \frac{c_{v}}{\nu^{8}} .
$$

Hence, any of the coefficient formulae for general Dirichlet series applied to coefficients with $\nu \leqslant N-B$ eliminates the $e_{\gamma}$ 's at once and gives an integral representation of

$$
\sum_{\substack{v \leqslant N-B \\(v, B)=1}} h(v) \Lambda(\nu+B)
$$

which, in very general cases, for $N \rightarrow \infty$ is

$$
\sim \sum_{B<p \leqslant N} \log p \cdot h(p-B) \sim \log N \sum_{(p, x)} 1
$$

where the last sum represents obviously the number of solutions of

$$
p=f\left(x_{1}, \ldots, x_{l}\right)+B, \quad p \leqslant N .
$$

Since-if we use appropriate coefficient formulae-the singularities of the integrand are among those of the $G_{B}(s, k, \chi)$-functions which are in turn, in very general cases, meromorphic on the whole plane, the contour-integration technique is fully applicable. This description of our general sieving process does not contain (1.4) literally, but it would be very easy to modify it so as to include (1.4) in it. We shall apply also this method systematically in later papers of this series; here we shall confine ourselves to prove the following
THEOREM I. For the number $\pi_{D}(N)$ of prime pairs $\left(p_{1}, p_{2}\right)$ with

$$
p_{1}-p_{2}=D, \quad p_{2} \leqslant N, \quad(D \text { even })
$$

the representation

$$
\text { (1.11) } \begin{aligned}
\pi_{D}(N)=\{1+O(\varepsilon(N))\} & B_{1} \frac{N}{\log ^{2} N} \prod_{\substack{p \mid B \\
p>2}} \frac{p-1}{p-2}- \\
& -\frac{1+O(1 / \sqrt{\log N)}}{\log ^{2} N} \sum_{\substack{k \leq N+D) \\
(k, D)=1}} \frac{\mu(k) \log (N / k)}{\varphi(k)} \times \\
& \times \sum_{\chi \bmod k} \bar{\chi}(-D, k) \sum_{|\gamma|<\log N \cdot \exp (3 / 2)(N))}\left\{\frac{N^{e}-N^{e / 100}}{\varrho(1+\varrho / \log N)^{[\varepsilon(N) \log N]+1}}\right\}
\end{aligned}
$$

holds.

Here $B_{1}$, the Hardy-Littlewood constant in $(1.2), D \leqslant \frac{N}{\log ^{10} N}, \varepsilon(x)$ is positive and tends to 0 with $1 / x$ arbitrarily slowly but so that

$$
\varepsilon(x) \sqrt{\log x} \rightarrow \infty \text {. }
$$

The $O$-sign refers to $N \rightarrow \infty$ uniformly in $D$.

This explicit representation holds of course without conjectures. In the main term one can recognise at once the heuristical Hardy-Littlewood formula in (1.1). One could prove also here (somewhat less strongly than before) that one could drop from the critical sum in (1.11) everything except the contribution of the zeros "near to $\sigma=\frac{1}{2}$ ". Theorem I of this paper (and the theorem in paper [3]) seem to be the first ones of this type in the literature. Another thing which gives significance to such explicit formulae "of Riemann type" is the fact that several problems can be reduced to averaging of (1.11) with respect to $D$ and owing to the structure of the formula (1.11) this leads to character-sums non-trivially estimable. Since after the relation (1.4) (and some facts from the proof of Theorem II) the proof of Theorem I is easy, we shall postpone it to an appendix.

2. As I observed recently (see [4]), another function-theoretical sieve-method can be devised to investigate "definite" problems of the additive prime number theory typical of them being the binary Goldbach problem or the representability of the integer $N$ in the form $p+x_{1}^{2}+x_{2}^{2}$. As given in [4] it is possible to construct a function which can be developed into Dirichlet series in two ways, both convergent (even absolutely) in a halfplane $\sigma>0$ the corresponding coefficients being thus equal. In one series the coefficient of $N^{-8}$ is "essentially" the number of different representations Acta Arithmetica Xrri.1 
of $N$ in the required form, in the other series the corresponding coefficient is an asymptotical representation of it.

In particular, as regards the binary Goldbach problem, I stated l.c. without proof a theorem which in a slightly more general form runs as follows

THEOREM II. Let $M / 2 \leqslant N \leqslant M\left(N\right.$ even) and $v_{2}(N)$ stand for the number of binary Goldbach decompositions of the even $N$. Then the "explicit" formula

$$
\begin{aligned}
& \text { (2.1) } \quad \nu_{2}(N)=\{1+O(\varepsilon(N))\} \frac{N}{\log ^{2} N} B_{1} \prod_{\substack{p ! N \\
p>2}} \frac{p-1}{p-2}- \\
& -\frac{1+O(1 / \sqrt{\log N})}{\log ^{2} M} \sum_{\substack{k \leq M M \\
(k, N)=1}} \frac{\mu(k) \log (M / k)}{\varphi(k)} \times \\
& \times \sum_{z \bmod k} \bar{x}(N, k) \sum_{|\gamma|<\log M \exp (3 / 2 \varepsilon(M))} \frac{N^{\varrho}-N^{\text {re/100 }}}{\varrho(1+\varrho / \log M)^{[\varepsilon(M) \log M]+1}}
\end{aligned}
$$

holds $\left({ }^{3}\right)$.

Here again $B_{1}$ is the Hardy-Littlewood constant-in (1.2) and $\varepsilon(x)$ is positive and tends to 0 with $1 / x$ arbitrarily slowly but so that

$$
\varepsilon(x) \sqrt{\log x} \rightarrow \infty
$$

The $O$-sign refers of course to $M \rightarrow \infty$.

In the main term one recognises again the heuristic formula for the number of the binary Goldbach decompositions given by Hardy and Littlewood in their paper [1] (in particular in p. 32, Conjecture A). It is perhaps not uninteresting to note in advance that "the singular factors"

$$
B_{1} \prod_{p \mid D, p>2} \frac{p-1}{p-2} \quad \text { resp. } \quad B_{1} \prod_{p|| N, p>2} \frac{p-1}{(p)} \frac{p-2}{p-2}
$$

in our theorems do not arise from singular series in Hardy-Littlewood's sense; no dissection of the line of integration occurs at all. Also such Riemann type explicit formula for the Goldbach problem seems to me unnoticed so far. Here the assertion that this formula gives a clearer insight into the essence of the problem is even more plausible than before since in several important problems "the averaging with respect to $N$ " means for our critical sum estinuation of character-sums, sometimes with consecutive integer arguments, for "long" intervals which can be performed

( $\left.{ }^{3}\right)$ The theorem could have been stated replacing everywhere $M$ by $N$; however for "averaging" purposes this form is more suitable. non-trivially. We shall give here a detailed proof of Theorem II without referring to the general frame of the method in [4], and return to its. applications in subsequent papers of this series.

In accordance to what was said on the twin prime problem also here can be asserted that the truth of the (binary) Goldbach conjecture depends exclusively on the "small" zeros of $L(s, k, \chi)$ functions notably on those "near" to the line $\sigma=\frac{1}{2}$. This follows from a suitable modification of Theorem II and we shall return to it in a later paper of this series.

3. For the proofs of our theorems we shall need a number of auxiliary propositions. Let

$$
\begin{gathered}
\alpha>1, \quad x>0, \quad \omega \geqslant 3, \quad \delta \geqslant c(>14) \text { and integer } \\
\Phi(x, \delta, \omega)=\Phi(x) \stackrel{\text { def }}{=} \frac{1}{2 \pi i} \int_{(\alpha)} \frac{x^{w}}{w(1+w / \omega)^{\delta+1}} d w .
\end{gathered}
$$

Besides the evident relation

$$
\Phi(x)=0 \quad \text { for } \quad 0 \leqslant x \leqslant 1
$$

we need

LEMMA I. For all $x \geqslant 0 \Phi(x)$ is non-decreasing and

(a) $0 \leqslant \Phi(x)<1$,

(b) for $x \geqslant \exp (2 \delta / \omega)$ we have

$$
1-\exp (-\delta / 5) \leqslant \Phi(x) \quad(<1)
$$

(c) supposing for integer $N>0$ also $\omega \leqslant \log 2 N$ we have

$$
\Phi\left(\frac{N}{N-1}\right)<N^{-2} \text {. }
$$

For the proof we remark that for $x \geqslant 1$ we have

$$
\text { (3.4) } \Phi(x)=1+\frac{\omega^{\delta+1}}{\delta !}\left(x^{w} w^{-1}\right)_{w=-\omega}^{(\delta)}=1-\exp (-\omega \log x) \sum_{\nu=0}^{\delta} \frac{(\omega \log x)^{\nu}}{\nu !} \text {. }
$$

Since we have for all real $r$ s

$$
1-e^{-r} \sum_{\nu=0}^{\delta} \frac{r^{\nu}}{\nu !}=\frac{1}{\delta !} \int_{0}^{r} e^{-\lambda} \lambda^{\delta} d \lambda
$$

(3.4) gives for $x \geqslant 1$

$$
\Phi(x)=\frac{1}{\delta !} \int_{0}^{\omega \log x} e^{-\lambda} \lambda^{\delta} d \lambda
$$


which, using also the relation $\Phi(x)=0$ for $0 \leqslant x \leqslant 1$, puts the monotonicity and (a) into evidence. In case (b) we have

$\omega \log x \geqslant 2 \delta$

and thus

$$
\begin{aligned}
(0<) 1-\Phi(x) & =\frac{1}{\delta !} \int_{\omega \log x}^{\infty} e^{-\lambda} \lambda^{\delta} d \lambda \\
& \leqslant \frac{1}{\delta !} \int_{2 \delta}^{\infty} e^{-\lambda} \lambda^{\delta} d \lambda=\frac{\delta^{\delta+1}}{\delta ! e^{\delta}} \int_{1}^{\infty}\left\{(1+r) e^{-r}\right\}^{\delta} d r .
\end{aligned}
$$

Since for $r \geqslant 1$ we have

$$
\log (1+r) \leqslant r \log 2, \quad(1+r) e^{-r} \leqslant e^{-r / 4}
$$

we get

$$
1-\Phi(x) \leqslant 4 \frac{\delta^{\delta}}{\delta !} \exp \left(-\frac{5}{4} \delta\right)<2 \exp \left(-\frac{\delta}{4}\right)<\exp \left(-\frac{\delta}{5}\right)
$$

which proves (b). Finally from (3.5) (roughly) indeed

$$
\Phi\left(\frac{N}{N-1}\right)<\delta !^{-1} \int_{0}^{\frac{\log 2 N}{N-2}} \lambda^{\delta} d \lambda<N^{\frac{-\delta+1}{2}}<N^{-2} .
$$

4. Let $N>c$, further

and

$$
1 \geqslant \lambda \geqslant 1-\frac{1}{\log \log N}
$$

$$
f(m, \lambda) \stackrel{\text { def }}{=} \sum_{p \mid m}(p) p^{-\lambda}
$$

the summation being extended to the different prime factors of $m$ only. Then we need the simple

LEMMA II. The inequality

holds.

$$
\max _{1 \leqslant m \leqslant N} f(m, \lambda)<6 \log \log \log N
$$

For the proof we remark first that the maximum in question is obviously attained also for a square-free $m=m_{0}$ and further this $m_{0}$ has evidently the form

$$
m_{0}=2 \cdot 3 \cdot 5 \cdot 7 \cdot 11 \cdot \ldots \cdot p_{r}
$$

where $r$ is defined by

$$
2 \cdot 3 \cdot \ldots \cdot p_{r} \leqslant N<2 \cdot 3 \cdot \ldots \cdot p_{r_{+1}} \cdot
$$

Since for $N>c$ we have as well-known

$$
\frac{1}{2} \log N \leqslant p_{r} \leqslant 2 \log N, \quad \frac{1}{2} \cdot \frac{\log N}{\log \log N} \leqslant r \leqslant 2 \frac{\log N}{\log \log N}
$$

and for $x>c$

$$
\pi(x)<\frac{2 x}{\log x}
$$

we get for $N>c$

$$
\begin{aligned}
\max _{1 \leqslant m \leqslant N} f(m, \lambda) & =\sum_{p=1}^{r} p_{v}^{-\lambda}=\int_{3 / 2}^{p_{r}} x^{-\lambda} d \pi(x)=r p_{r}^{-\lambda}+\lambda \int_{3 / 2}^{p_{r}} \pi(x) x^{-\lambda-1} d x \\
& <4 \frac{\log ^{1-\lambda} N}{\log \log N}+2 \int_{3 / 2}^{p_{r}} \frac{x^{1-\lambda}}{x \log x} d x<O(1)+2 e \log \log \log N \\
& <6 \log \log \log N
\end{aligned}
$$

indeed.

Putting with the above $\lambda$

$$
f_{0}(m, \lambda) \stackrel{\text { def }}{=} \sum_{p \mid m} \frac{\log p}{p^{\lambda}-1}
$$

(summation extended again only over the different prime factors) we get for $N>c$ by Lemma II,

$$
\max _{1 \leqslant m \leqslant N} f_{0}(m, \lambda) \leqslant 3 \log N \max _{1 \leqslant m \leqslant N} f(m, \lambda)<\log ^{2} N .
$$

Defining further for $z=x+i y$ the function $D(m, z)$ by

$$
D(m, z) \stackrel{\text { def }}{=} \prod_{p \mid m} \frac{1}{1-1 / p^{z}}
$$

(product extended only to different prime factors of $m$ ) we get from Lemma II for $N>c$ and

$$
1-\frac{1}{\log \log N} \leqslant x \leqslant 2
$$


the inequality (4.7) $\max _{1 \leqslant m \leqslant N}|D(m, z)| \leqslant \max _{1 \leqslant m \leqslant N} D(m, x) \leqslant c \max _{1 \leqslant m \leqslant N} \exp f(m, x)<c(\log \log N)^{6}$. $1 \leqslant m \leqslant N$

Further we have

$$
\left|\frac{d D(m, z)}{d z}\right|=|D(m, z)|\left|\sum_{p \mid m} \frac{\log p}{p^{z}-1}\right| \leqslant|D(m, z)| f_{0}(m, x)
$$

and hence, in the range (4.6), using (4.4) and (4.7), we get for $N>c$

$$
\max _{1 \leqslant m \leqslant N}\left|\frac{d D(m, z)}{d z}\right| \leqslant \log ^{3} N
$$

5. We shall need further

LFMrMa III. For $1 \leqslant m \leqslant M$ integer, $m$ even, we have $\left({ }^{4}\right)$ for $M>0$ the inequality $\left(B_{1}\right.$ in (1.2))

$$
\left|\sum_{\substack{1 \leq k \leq M \\(k, m)=1}} \frac{\mu(k) \log (M / k)}{\varphi(k)}-B_{1} \prod_{\substack{p \mid m \\ p>2}} \frac{p-1}{p-2}\right|<c \log ^{-16} M .
$$

For the proof we consider the function $\varphi_{m}(z)$ defined for $x>0$ by

$$
\sum_{(k, m)=1} \frac{\mu(k)}{\varphi(k)} k^{-z}
$$

This can be written as

$$
\prod_{(p, m)=1}\left\{1-\frac{p^{-z}}{p-1}\right\}
$$

and hence

$\varphi_{m}(z)=\frac{1}{L\left(z+1, m, \chi_{0}\right)} \prod_{(p, m)=1}\left\{1-\frac{1 /(p-1) p^{z+1}}{1-1 / p^{z+1}}\right\}=\frac{1}{L\left(z+1, m, \chi_{0}\right)} U_{1}(z)$

where $U_{1}(z)$ (and later $U_{2}(z), \ldots$ ) is regular for, say, $x \geqslant-\frac{1}{t}$, and here the inequality

$$
\frac{1}{c} \leqslant\left|U_{v}(z)\right| \leqslant c
$$

${ }^{(4)}$ If $m$ is odd the product must be replaced by 0 , as is clear from the proof we shall not need it. We put no stress in obtaining a possibly good error-term. holds. (5.1) and (5.2) give for $x>0$

$$
\begin{aligned}
(5.4)- & \sum_{(k, m)=1} \frac{\mu(k) \log k}{\varphi(k)} k^{-z} \\
& =\varphi_{m}^{\prime}(z)=\varphi_{m}(z)\left(\log \varphi_{m}(z)\right)^{\prime} \\
& =\frac{U_{1}(z)}{L\left(z+1, m, \chi_{0}\right)}\left\{U_{2}(z)-\frac{L^{\prime}}{L}\left(z+1, m, \chi_{0}\right)\right\} \\
& =U_{1}(z)\left(\frac{1}{L\left(z+1, m, \chi_{0}\right)}\right)^{\prime}+\frac{U_{3}(z)}{L\left(z+1, m, \chi_{0}\right)} \\
& =U_{1}(z)\left(\frac{1}{\zeta(z+1)} \prod_{p \mid m} \frac{1}{1-1 / p^{z+1}}\right)^{\prime}+\frac{U_{3}(z)}{\zeta(z+1)} \prod_{p \mid m} \frac{1}{1-1 / p^{z+1}} \\
& =-U_{1}(z) \frac{\zeta^{\prime}(z+1)}{\zeta(z+1)^{2}} D(m, z+1)+\frac{U_{1}(z)}{\zeta(z+1)} \cdot \frac{d D(m, z+1)}{d z}+ \\
& +\frac{U_{3}(z)}{\zeta(z+1)} D(m, z+1) \stackrel{\text { def }}{=} g(z) .
\end{aligned}
$$

We remark further that ( $m$ being even)

(5.5)

$U_{1}(0) \prod_{p \mid m} \frac{1}{(p)}=\prod_{(p, m)=1}\left\{1-\frac{1}{(p-1)^{2}}\right\} 2 \prod_{\substack{p \mid m \\ p>2}} \frac{p}{p-1}=B_{1} \prod_{\substack{p \mid m \\ p>2}} \frac{p-1}{p-2}$.

Starting from the integral

$$
J_{1}=\frac{1}{2 \pi i} \int_{1 / \log M-i \log ^{20} M}^{1 / \log M+i \log ^{20} M} \frac{\left(M+\frac{1}{2}\right)^{z}}{z} g(z) d z,
$$

the series representation of $g(z)$ in (5.4) gives

$$
\begin{aligned}
J_{1} & =-\sum_{(k, m)=1} \frac{\mu(k) \log k}{\varphi(k)} \cdot \frac{1}{2 \pi i} \int_{1 / \log M-i \log ^{20} M}^{1 / \log M+i \log ^{20} M} \frac{1}{z}\left(\frac{M+\frac{1}{2}}{k}\right)^{z} d z \\
& =-\sum_{k \leqslant M,(k, m)=1} \frac{\mu(k) \log k}{\varphi(k)}+O\left(\log ^{-20} M\right) \sum_{(k)} \frac{\log k}{\varphi(k)} \cdot \frac{k^{-1 / \log M}}{\log \frac{M+\frac{1}{2}}{k}}
\end{aligned}
$$


and hence routine reasoning gives

$$
J_{1}=-\sum_{\substack{k \leqslant M \\(k, m)=1}} \frac{\mu(k) \log k}{\varphi(k)}+O\left(\log ^{-17} M\right) .
$$

Let further be

$$
J_{2}=\frac{\log M}{2 \pi i} \int_{1 / \log M-i \log ^{20} M}^{1 / \log M+i \log ^{20} M} \frac{\left(M+\frac{1}{2}\right)^{z}}{z} \varphi_{m}(z) d z .
$$

(5.1) gives again as before

$$
J_{2}=\sum_{\substack{k \leqslant M \\(k, m)=1}} \frac{\mu(k)}{p(k)} \log M+O\left(\log ^{-16} M\right)
$$

and hence

$$
J_{1}+J_{2}=\sum_{\substack{k \leqslant M \\(k, m)=1}} \frac{\mu(k) \log ^{\prime}(M / k)}{\varphi(k)}+O\left(\log ^{-16} M\right) .
$$

Using the representations (5.2) resp. (5.4), we can apply Cauchy's theorem to the parallelogramm with the vertices

$$
\frac{1}{\log M} \pm i \log ^{20} M, \quad-\frac{1}{\log \log M} \pm i \log ^{20} M
$$

Since this parallelogramm lies for $M>c$ in the zerofree domain of $L\left(z+1, m, \chi_{0}\right)$, the only singularity in the inside is the pole of first order of $g(z)$ at $z=0$ with the residuum

$$
g(0)=U_{1}(0) \prod_{p \mid m} \frac{1}{(p)}=B_{1} \prod_{\substack{p \mid m \\ p>2}} \frac{p-1}{1-1 / p}
$$

using (5.5). As is well known, on the periphery of our parallelogramm we have for $M>c$

$$
\left|\frac{\zeta^{\prime}(z+1)}{\zeta(z+1)^{2}}\right|<(\log \log M)^{c}, \quad \frac{1}{|\zeta(z+1)|}<(\log \log M)^{c} ;
$$

these, combined with (4.5), (4.6), (4.7) and (4.8), give for the remaining parts of the contourintegral the upper bound

$$
O\left(\log ^{-20} M\right) \log ^{4} M+O\left(M^{-1 / \log \log M}\right) \log ^{5} M
$$

which completes the proof of Lemma III.
We shall further need the

LEMMA IV $\left({ }^{5}\right)$. For square-free odd $k$ the estimation

$$
Z(m, k) \stackrel{\text { def }}{=}\left|\sum_{\chi \bmod k}^{*} \chi(m, k)\right| \leqslant(m-1, k)
$$

holds.

For the proof we remark that if

$$
k=p_{1} p_{2} \ldots p_{l}, \quad p_{1}<p_{2}<\ldots<p_{l},
$$

$g_{j}$ is a primitive root $\bmod p_{j}$ and, further, if

$$
m \equiv g_{j}^{\xi} \bmod p_{j}, \quad 0 \leqslant \xi_{j} \leqslant p_{j}-2, \quad j=1, \ldots, l
$$

then for fixed $v_{j}$ 's with

$$
0 \leqslant v_{j} \leqslant p_{j}-2, \quad j=1,2, \ldots, l
$$

the characters belonging to $\bmod k$ are given by

$$
\chi(m, k)=\exp \left\{2 \pi i\left(\frac{\nu_{1} \xi_{1}}{p_{1}-1}+\ldots+\frac{\nu_{l} \xi_{l}}{p_{l}-1}\right)\right\} .
$$

Since they are primitive characters if and only if

$$
1 \leqslant v_{j} \leqslant p_{j}-2, \quad j=1,2, \ldots, l,
$$

we indeed have

$$
\begin{aligned}
Z(m, k) & =\prod_{j=1}^{l} Z\left(m, p_{j}\right)=\prod_{j=1}^{l}\left|\sum_{j_{j}=1}^{p_{j}-2} \exp \frac{2 \pi i v_{j} \xi_{j}}{p_{j}-1}\right| \\
& =\prod_{\xi_{j}=0}\left(p_{j}-2\right)=\prod_{p_{j} \mid(m-1)}\left(p_{j}-2\right)<\prod_{\eta_{j} \mid(m-1)} p_{j}=!(m-1, k) \mid .
\end{aligned}
$$

6. Now we turn to the proof of Theorem II. Iet $M>c$, further, let $\omega, \omega_{1}$ and $\delta$ be functions of $M$ to be determined later; here we restrict them only by
(6.4)
$3 \leqslant \omega \leqslant \log M$,
$3 \log \log M \leqslant \omega_{1} \leqslant 0,99 \log M$, $\delta \geqslant \sqrt{\log M}$ and integer,

$$
\delta / \omega=o(1)
$$

and for the even $N$$$
M / 2 \leqslant N \leqslant M
$$

(5) This lemma was also needed in [4]; we include it for the reader's convenience. 
Let $s=\sigma+i t, \sigma>2$, further, let $\eta$ be such that

(6.6)

$$
1<\eta<\sigma-1
$$

we start with the function

(6.7) $\quad F_{M}(s)=-\sum_{k \leqslant M} \frac{\mu(k) \log (M / k)}{\varphi(k)} \times$

$$
\times\left\{\sum_{\chi \bmod k} \frac{1}{2 \pi i} \int_{(\eta)} L(s-w, k, \bar{\chi}) \frac{L^{\prime}}{L}(w, k, \chi) \frac{1-e^{-\omega_{1} w}}{w(1+w / \omega)^{\delta+1}} d w\right\} .
$$

Owing to $(6.6)$ we can replace both $L(s-w, k, \bar{\chi})$ and $\frac{L^{\prime}}{L}(w, k, \chi)$ by their respective Dirichlet series and integrate termwise. This gives

$$
\text { (6.8) } \begin{aligned}
F_{M}(s) & =\sum_{k \leqslant M} \mu(k) \log (M / k) \sum_{(n, k)=(v, k)=1} \sum_{(v)} \frac{\Lambda(\nu)}{n^{s}} \times \\
\times\left\{\frac{1}{\varphi(k)} \sum_{\chi \bmod k}(x)\right. & \chi(v, k) \bar{\chi}(n, k)\} \frac{1}{2 \pi i} \int_{(\eta)} \frac{(n / v)^{w}-\left(n e^{-\omega} / v\right)^{w}}{w(1+w /(1))^{\delta+1}} d w .
\end{aligned}
$$

The last integral can be evaluated by using Lemma I; what we actually need is only that it is

$$
\text { (a) }=0 \text { for } v \geqslant n
$$

(6.9) (b) between 0 and 1 for $v<n$

(c) between $1-\exp \left(-\frac{1}{5} \delta\right)$ and 1 for $n e^{-\omega_{1}} \leqslant \nu \leqslant n \exp (-2 \delta / \omega)$.

Denoting the value of the integral in $(6.8)$ by $b(n, v)$, we get from $(6.8)$

$$
F_{M}(s)=\sum_{(n)} \frac{a_{n}}{n^{s}}
$$

where

$$
a_{n}=\sum_{\substack{k \leq M \\(k, n)=1}} \mu(k) \log (M / k) \sum_{\substack{\nu<n \\(\nu, k)=1 \\ n \equiv \nu \bmod k}} \Lambda(\nu) b(n, \nu)
$$

This representation shows at once that the series (6.10) converges (even absolutely) in a half-plane $\sigma>c$.

7. We investigate in particular $a_{N}$ (6.11) gives, taking also in account Lemma I (c)

(7.1) $\quad a_{N}=\sum_{v \leqslant N-2} \Lambda(v) b(N, v) \sum_{\substack{k, N)=(k, v)=1 \\ k(N-v)}} \mu(k) \log (M / k)+O(1 / M)$, which can be written as $\left(a_{N}^{\prime}+a_{N}^{\prime \prime}\right)$, where

$$
\begin{aligned}
& \text { (7.2) } \quad a_{N}^{\prime}=\sum_{\substack{\nu \leqslant N-2 \\
(v, N)=1}} \Lambda(\nu) b(N, v) \sum_{\substack{(k, N)=(k, v)=1 \\
k\}(N-v)}} \mu(k) \log (M / k) \text {, } \\
& \text { (7.3) } \quad a_{N}^{\prime \prime}=\sum_{\substack{\nu, N \\
(v, N)>1}} \Lambda(v) b(N, v) \sum_{\substack{(k, N)=(k, v)=1 \\
k \mid(N), v)}} \mu(k) \log (M / k)+O(1 / M) .
\end{aligned}
$$

We consider the inner sum in (7.2). Since owing to $(\nu, N)=1$ and $k \mid(N-\nu)$ the restrictions $(k, N)=(k, \nu)=1$ are automatically satisfied, this is owing to $N-v \geqslant 2$ equal to

$$
\sum_{k !(N-v)} \mu(k) \log (M / k)=\Lambda(N-\nu)
$$

and hence

$$
\text { (7.4) } \quad a_{N}^{\prime}=\sum_{\substack{v \leqslant N-2 \\(v, N)=1}} \Lambda(v) \Lambda(N-v) b(N, v) \text {. }
$$

As to $a_{N}^{\prime \prime}$ in (7.3), the inner sum cannot exceed absolutely (roughly)

$$
\log M \cdot \tau(N-\nu)
$$

and hence, using also (6.9)

$$
\left|a_{N}^{\prime \prime}\right|<2 \log M \max _{1 \leq l \leq M} \tau(l) \sum_{\substack{\nu \leq M \\(\nu, N)>1}} \Lambda(v)
$$

But this last sum is equal to

$$
\sum_{p \mid N} \log p \sum_{p^{\lambda} \leqslant M} 1=\sum_{p, N} \log p \cdot\left[\frac{\log M}{\log p}\right]<\log M \cdot \tau(N)
$$

and hence for $M>c$

$$
\left|a_{N}^{\prime \prime}\right|<2 \log ^{2} M \max _{l \leqslant M} \tau(l)^{2}<M^{1 / 10} .
$$

This and (7.4) give

$$
a_{N}=\sum_{\substack{p<N \\(v, N)=1}} \Lambda(v) \Lambda(N-v) b(N, v)+O\left(M^{1 / 10}\right) ;
$$

since the contribution of prime-powers $p^{\alpha}$ with $\alpha \geqslant 2$ cannot exceed $2 \log ^{2} M \cdot O(\sqrt{M} \log M)$, we get

and also

$$
a_{N}=\sum_{\substack{p_{1}+p_{2}=N \\\left(p_{j}, N\right)=1}} \log p_{1} \log p_{2} \cdot b\left(N, p_{1}\right)+O\left(\sqrt{M} \log ^{3} M\right)
$$


8. In order to get a more elegant form for $a_{N}$, we remark first that $(6.2)$ and $(6.9)(\mathrm{b})$ give at once

$$
\sum_{\substack{p_{1}+p_{2}=N \\ \min p_{j} \leqslant N e^{-\omega_{1}}}} \log p_{1} \log p_{2} \cdot b\left(N, p_{1}\right)<\log ^{2} N \cdot N e^{-\omega_{1}}<\frac{M}{\log M} .
$$

Further, according to Schnirelman's theorem $\left(^{6}\right)($ and $(6.9)(\mathrm{b})$ and $(6.4)$ )

$$
\begin{gathered}
\sum_{\substack{p_{1}+p_{2}=N \\
\max p_{j}>N \exp \left(-2 \frac{\delta}{\omega}\right)}} \log p_{1} \log p_{2} \cdot b\left(N, p_{\mathrm{q} 1}\right)<\log ^{2} N \sum_{\substack{p_{1}+p_{2}=N \\
\operatorname{manx} p_{j}>N \exp \left(-2 \frac{\delta}{\omega}\right)}} 1 \\
<c N \frac{\delta}{\omega} \prod_{p_{\mid} \mid N}\left(1+\frac{1}{p}\right)<c M \frac{\delta}{\omega} \prod_{\substack{p \mid N \\
p>2}} \frac{p-1}{p-2} .
\end{gathered}
$$

Replacing further in the remaining part of the sum in $(7.5) b\left(N, p_{1}\right)$ by 1 the error is, owing to (6.9)(c) and (6.3), easily

$$
e^{-\delta / 5} \log ^{2} N \cdot c \frac{N}{\log N}<c \frac{M}{\log ^{10} M} \prod_{\substack{p \mid N \\ p>2}} \frac{p-1}{p-2}
$$

Collecting all these we get

$$
\text { (8.4) } \quad a_{N}=\sum_{p_{1}+p_{2}=N} \log p_{1} \log p_{2}+O(M)\left(\frac{1}{\log M}+\frac{\delta}{\omega}\right) \prod_{\substack{p p_{1} N \\ p>2}} \frac{p-1}{p-2}
$$

9. Next we proceed to obtain another Dirichlet series for $F_{M}(s)^{\prime}$ convergent (even absolutely) in some half-plane $\sigma>c$. For a character $\chi(n, k)$, let $k^{*}$ be its conductor and $\chi^{*}\left(n, k^{*}\right)$ the corresponding primitive character; then for a square free $k$, as is well known,

$$
L(w, k, \chi)=L\left(w, k^{*}, \chi^{*}\right) \prod_{p \mid \frac{k}{k^{*}}}\left(1-\frac{\chi^{*}\left(p, k^{*}\right)}{p^{w}}\right)
$$

and hence

$$
\text { (9.1) } \frac{L^{\prime}}{L}(w, k, \chi)=\frac{L^{\prime}}{L}\left(w, k^{*}, \chi^{*}\right)+\sum_{p \mid \frac{k}{k^{*}}} \frac{\chi^{*}\left(p, k^{*}\right) \log p}{p^{w}-\chi^{*}\left(p, k^{*}\right)} \text {. }
$$

${ }^{(6)}$ See e.g. K. Prachar [2], p. 51, Satz 4.4. Actually we have used here a slightly stronger form of the theorem - one could prove it mutatis mutandis - namely that the number of binary Goldbach decompositions of $N$ with $\max p_{j}>N-y$ cannot exceed $c \frac{y}{\log ^{2} N} \prod_{\mid N}\left(1+\frac{1}{p}\right)$ if $N / 2 \geqslant y \geq N / v \sqrt{\log N}$.
Then we write

$$
F_{M}(s)=F_{M 1}(s)+F_{M 2}(s),
$$

where by (6.7) (with the notation of p. 63)

$$
\begin{aligned}
F_{M 1}(s)=- & \sum_{k \leqslant M M} \frac{\mu(k) \log (M / k)}{\varphi(k)} \sum_{k^{*} \mid k} \sum_{\left(k^{*}\right)} \sum_{\left.\chi^{*} \bmod k^{*}\right)}^{\prime^{*}} \frac{1}{2 \pi i} \times \\
& \times \int_{(\eta)} L(s-w, k, \bar{\chi}) \frac{L^{\prime}}{L}\left(w, k^{*}, \chi^{*}\right) \frac{1-e^{-\omega_{1} w}}{w(1+w / \omega)^{\delta+1}} d w
\end{aligned}
$$

and

$$
\begin{aligned}
& F_{M 2}(s)=-\sum_{k \leqslant M} \frac{\mu(k) \log (M / k)}{\varphi(k)} \sum_{k^{*} \mid k} \sum_{(k *)} \sum_{\chi^{*} \bmod \left(\chi^{*}\right)}^{*} \frac{1}{2 \pi i} \times \\
& \times \int_{(\eta)} L(s-w, k, \bar{\chi}) \frac{1-e^{-\omega_{1} w}}{w(1+w / \omega)^{\delta+1}}\left\{\sum_{p \mid \frac{k}{k^{*}}} \frac{\chi^{*}\left(p, k^{*}\right) \log p}{p^{w}-\chi^{*}\left(p, k^{*}\right)}\right\} d w .
\end{aligned}
$$

10. Let us consider $F_{M 2}(s)$ first. Writing the inner sum in brackets in the form

$$
\sum_{p \mid \frac{k}{k^{*}}} \log p \sum_{\lambda=1}^{\infty} \frac{\chi^{*}\left(p^{\lambda}, k^{*}\right)}{p^{\lambda w}}
$$

and putting it into (9.4), we get

$$
\begin{array}{r}
F_{M_{2}}(s)=-\sum_{k \leqslant M} \frac{\mu(k) \log (M / k)}{\varphi(k)} \sum_{k^{*} \mid k} \sum_{\left(k^{*}\right)} \sum_{x^{*} \bmod \left(k^{*}\right)}^{*} \sum_{p \mid \frac{k}{k^{*}}} \log p \sum_{\lambda=1}^{\infty} x^{*}\left(p^{\lambda}, k^{*}\right) \times \\
\times \sum_{(n, k)=1} \frac{\bar{\chi}(n, k)}{n^{s}} \cdot \frac{1}{2 \pi i} \int_{(\eta)} \frac{\left(n / p^{\lambda}\right)^{w}\left(1-e^{-\omega_{1} w}\right)}{w(1+w / \omega)^{\delta+1}} d w .
\end{array}
$$

This gives using (6.9)(a)

$$
F_{M 2}(s)=\sum_{(n)} \frac{e_{n}^{(2)}}{n^{s}}
$$

where

(10.2) $e_{n}^{(2)}=$

$=-\sum_{\substack{k \leq M \\ k, n)=1}} \frac{\mu(k) \log (M / k)}{\varphi(k)} \sum_{k^{*} \mid k} \sum_{\substack {\left(k^{*}\right) \\ \begin{subarray}{c}{p<n \\ p \mid \frac{k}{k^{*}}{ ( k ^ { * } ) \\ \begin{subarray} { c } { p < n \\ p | \frac { k } { k ^ { * } } } }\end{subarray}} \log p \sum_{p^{\lambda}<n} b\left(n, p^{\lambda}\right) \sum_{x^{*} \bmod \left(k^{*}\right)}{ }_{(\lambda)}^{*} \bar{\chi}\left(n p^{-\lambda}, k^{*}\right)$. 
This representation shows at once that the series in (10.1) converges (absolutely) in a half-plane $\sigma>c$. For the estimation of $\left|e_{N}^{(2)}\right|$ we apply Lemma IV. Owing to $\left(p, k^{*}\right)=1$ we have for all $q$ primes dividing $k^{*}$

$$
n p^{-\lambda}-1 \equiv 0 \bmod q \rightleftarrows n-p^{\lambda} \equiv 0 \bmod q
$$

and hence

(10.3) $\quad\left|e_{N}^{(2)}\right| \leqslant \log ^{2} M \sum_{\substack{k \leqslant M \\(k, N)=1}} \frac{|\mu(k)|}{\varphi(k)} \sum_{k^{*} \mid k} \sum_{\substack{p *) \\(k<N) \\ p \mid \frac{k}{k^{*}}}} \sum_{p^{\lambda}<N}\left(N-p^{\lambda}, k^{*}\right)$.

Introducing $k_{1}$ and $k_{2}$ as new summation variables by

$$
k^{*}=k_{1}, \quad k / k^{*}=k_{2}, \quad .
$$

we have

$$
k=k_{1} k_{2}, \quad\left(k_{1}, k_{2}\right)=1
$$

and hence from (10.3)

$$
\text { (10.4) } \begin{aligned}
\left|e_{N}^{(2)}\right| & \leqslant \log ^{2} M \sum_{\substack{\left.k_{1} k_{2} \leqslant M_{1}, k_{2}\right) \\
\left(k_{1} k_{2}, N\right)=1}} \frac{\left|\mu\left(k_{1}\right)\right|\left|\mu\left(k_{2}\right)\right|}{\varphi\left(k_{1}\right) \varphi\left(k_{2}\right)} \sum_{\substack{p<N \\
p \mid k_{2}}} \sum_{p(p)}\left(N-p^{\lambda}, k_{1}\right) \\
& \leqslant \log ^{2} M \sum_{k_{1} \leqslant M} \frac{\left|\mu\left(k_{1}\right)\right|}{\varphi\left(k_{1}\right)} \sum_{p<N} \sum_{\left.k^{\lambda}\right)}\left(N-p^{\lambda}, k_{1}\right) \sum_{\substack{k_{2} \leqslant M \\
k_{2}=0 \bmod p}} \frac{\left|\mu\left(k_{2}\right)\right|}{\varphi\left(k_{2}\right)} .
\end{aligned}
$$

The last sum is owing to $\left(p, k_{2} / p\right)=1$

$$
=\frac{1}{p-1} \sum_{\substack{k_{3} \leqslant M\left(k_{3}\right) \\\left(k_{3}, p\right)=1}} \frac{\left|\mu\left(k_{3}\right)\right|}{\varphi\left(k_{3}\right)}<\frac{c}{p} \sum_{k_{3} \leqslant M} \frac{\log \log M}{k_{3}}<c \frac{\log ^{3 / 2} M}{p}
$$

and hence from (10.4)

$$
\begin{aligned}
\left|e_{N}^{(2)}\right| & \leqslant c \log ^{4} M \sum_{k_{1} \leqslant M} \frac{1}{\left(k_{1}\right)} \frac{1}{k_{1}} \sum_{p<N} \sum_{p} \frac{\left.(N) p^{\lambda}, k_{1}\right)}{p} \\
& \left.=c \log ^{4} M \sum_{p^{\lambda}<N} \frac{1}{p} \sum_{p^{\lambda}<N} \sum_{(\lambda)} \sum_{k_{1} \leqslant M} \frac{\left(N-k_{1}\right)}{k_{1}} . p_{1}\right)
\end{aligned}
$$

Again the last sum cannot exceed

$$
\sum_{d \mid\left(N-p^{\lambda}\right)} d \sum_{k_{4} \leqslant M(d)} \frac{1}{d k_{4}}<\log M \cdot \tau\left(N-p^{\lambda}\right)<\exp \left(c \frac{\log M}{\log \log M}\right)
$$

and thus

$$
\text { (10.5) }\left|e_{N}^{(2)}\right| \leqslant \exp \left(c \frac{\log M}{\log \log M}\right) \sum_{p<N} \frac{1}{p}\left[\frac{\log N}{\log p}\right]<\exp \left(c \frac{\log M}{\log \log M}\right) \text {. }
$$

11. Next we investigate the function $F_{M_{1}}(s)$ in (9.3). Shifting the line of integration to the line $\operatorname{Re} w=-0,99$, we get

$$
F_{M 1}(s)=F_{M 3}(s)+F_{M 4}(s),
$$

where $F_{M 3}(s)$ consists of the residual terms and

(11.2) $\quad F_{M 4}(s)=-\sum_{k \leqslant M} \frac{\mu(k) \log (M / k)}{\varphi(k)} \times$

$\times \sum_{k^{*} \mid k} \sum_{\left(k^{*}\right)}^{\gamma_{z^{*} \bmod }^{*}} \frac{1}{2 \pi i} \int_{(-0,99)} L(s-w, k, \bar{\chi}) \frac{L^{\prime}}{L}\left(w, k^{*}, \chi^{*}\right) \frac{1-e^{-\omega_{1} w}}{w(1+w / \omega)^{\delta+1}} d w$.

Replacing $L(s-w, k, \bar{\chi})$ by its Dirichlet series, we get

where

$$
\text { (11.4) } \begin{aligned}
& e_{n}^{(4)}=\sum_{\substack{k \leq M \\
(k, n)=1}} \frac{\mu(k) \log (M / k)}{\varphi(k)} \times \\
& \times \sum_{k^{*} \mid k} \sum_{(k *)} \sum_{\chi^{*} \bmod \left(k^{*}\right)}^{*} \bar{\chi}(n, k) \frac{1}{2 \pi i} \int_{(-0,99)} \frac{L^{\prime}}{L}\left(w, k^{*}, \chi^{*}\right) \frac{n^{w}\left(1-e^{-\omega_{1} w}\right)}{w(1+w / \omega)^{\delta+1}} d w .
\end{aligned}
$$

Trivial estimation shows again that the series (11.3) converges (absolutely) in a half-plane $\sigma>c$. In particular (putting $w=-0,99+i v$ ), we get

$$
\begin{aligned}
& \text { (11.5) }\left|e_{N}^{(4)}\right| \leqslant 2\left(\frac{e^{\omega_{1}}}{N}\right)^{0,99} \log M \sum_{k \leqslant M} \frac{1}{(k)} \times \\
& \times \sum_{k^{*} \mid k}\left(k^{*}\right) \\
& x_{x^{*} \bmod \left(k^{*}\right)}^{*} \frac{1}{\pi} \int_{0}^{\infty}\left|\frac{L^{\prime}}{L}\left(-0,99+i v, k^{*}, \chi^{*}\right)\right| \frac{d v}{\left|\frac{1}{2}+i v\right|\left|1-\frac{1}{\omega}+i \frac{v}{\omega}\right|^{\delta+1}} .
\end{aligned}
$$

Splitting up the range of the integral into

$$
\int_{0}^{1}+\int_{i}^{\omega^{2}}+\int_{\omega^{2}}^{\infty}
$$


we obtain respectively

$$
\begin{gathered}
\int_{0}^{1}<c \log M \\
\int_{1}^{\omega^{2}}<c \int_{1}^{\omega^{2}} \frac{\log k^{*}(2+v)}{v} d v<c \log M \cdot \log ^{2} \omega<c \log ^{3} M
\end{gathered}
$$

and

$$
\int_{\omega^{2}}^{\infty}<e \int_{\omega^{2}}^{\infty} \frac{\log k^{*} v}{v^{2}} d v<c \log M
$$

hence, using also (6.2) and (6.1),

$$
\left|e_{N}^{(4)}\right| \leqslant c M^{0,9999} \log ^{4} M<e \frac{M}{\log M} .
$$

12. Now we consider $F_{M 3}(s)$ from (11.1). The residual terms are due to poles at

$$
w=1, \quad w=0, \quad w=\varrho
$$

(see the footnote on p.63). The contribution of poles at $w=1$ is obviously from (9.3)

(12.1) $\frac{1-e^{-\omega_{1}}}{(1+1 / \omega)^{\delta+1}} \sum_{k \leqslant M} \frac{\mu(k) \log (M / k)}{\varphi(k)} L\left(s-1, k, \chi_{0}\right) \stackrel{\text { dof }}{=} F_{M 3}^{(1)}(s)$.

This can be written as

with

$$
e_{n}^{(3,1)}=\frac{1-e^{-\omega_{1}}}{(1+1 / \omega)^{\delta+1}} n \sum_{\substack{k \leq M \\(k, n)=1}} \frac{\mu(k) \log \left(M / k_{i}\right)}{\varphi(k)},
$$

which shows at once that the series in (12.2) converges (absolutely) for $\sigma>c$. In particular, for $n=N$ Lemma III gives

$$
e_{N}^{(3,1)}=B_{1} N \frac{1-e^{-\omega_{1}}}{(1+1 / \omega)^{\delta+1}} \prod_{\substack{p|N| \\ p>2}} \frac{p-2}{p-1}+O\left(\frac{M}{\log M}\right)
$$

and thus from (6.2) and (6.4)

$$
e_{N}^{(3,1)}=\left\{1+O\left(\frac{\delta}{\omega}\right)+O\left(\frac{1}{\log M}\right)\right\} B_{1} N \prod_{\substack{p \mid N \\ p>2}} \frac{p-1}{p-2} .
$$

13. Next we consider the contribution of the poles at $w=0$. Ther occur only if $k^{*}>1$ and $\chi^{*}\left(-1, k^{*}\right)=+1$; hence the contribution of these poles is from (9.3)

(13.1) $\quad-\omega_{1} \sum_{k \leqslant M} \frac{\mu(k) \log (M / k)}{\varphi(k)} \sum_{\substack{k^{*} \neq k \\ k^{*}>1}} \sum_{\left(k^{*}\right)} \sum_{\substack{* \\ x^{*}\left(-1, k^{*}\right)=+1}}^{*}\left(_{\left(x^{*}\right)}^{*} L(s, k, \bar{x}) \stackrel{\text { def }}{=} F_{M 3}^{(2)}(s)\right.$,

which can be written as

$$
\sum_{(n)} \frac{e_{n}^{(3,2)}}{n^{s}}
$$

with

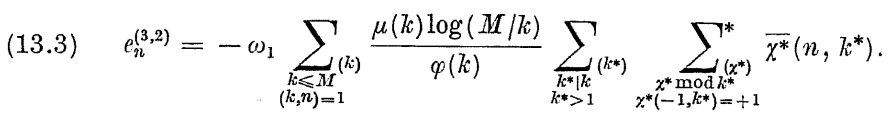

This again shows at once that series (13.2) converges (absolutely) in a half-plane $\sigma>c$. Considering again $e_{N}^{(3,2)}$, the inner sum in (13.3) can be written as $\left({ }^{7}\right)$

$$
\sum_{\left.x^{*} \bmod k^{*}\right)}^{*} \chi^{*}\left(N, k^{*}\right) \frac{1+\chi^{*}\left(-1, k^{*}\right)}{2}
$$

and hence its absolute value cannot exceed (in the notation of Lemma IV)

$$
\frac{1}{2}\left(Z\left(N, k^{*}\right)+Z\left(-N, k^{*}\right)\right) \text {. }
$$

This shows-also by $(6.2)-$

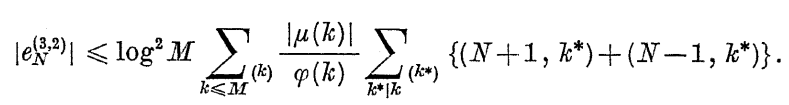

But since

$$
\sum_{k^{*} \mid k}\left(k^{*}\right)\left(N \pm 1, k^{*}\right) \leqslant(N \pm 1, k) \tau(k),
$$

we get

$$
\left|e_{N}^{(3,2)}\right| \leqslant \exp \left(c \frac{\log M}{\log \log M}\right) \sum_{k \leqslant M} \frac{|\mu(k)|}{\varphi(k)}\{(N+1, k)+(N-1, k)\} .
$$

Since

$$
\begin{aligned}
& \sum_{k \leqslant M} \frac{|\mu(k)|}{\varphi(k)}(N \pm 1, k) \leqslant \sum_{d_{l}(N \pm 1)} \frac{d}{\varphi(d)} \sum_{r \leqslant M \mid d} \frac{|\mu(r)|}{\varphi(r)} \\
& <c(\log \log M)^{2} \sum_{d !(N \pm 1)} \sum_{r \leqslant M} \frac{1}{r} \frac{1}{r}<\exp \left(c \frac{\log M}{\log \log M}\right),
\end{aligned}
$$

( $\left.{ }^{7}\right)$ Simplified by Mr. I. Kátai and Mr. I. Környei. 
we get

$$
\left|e_{N}^{(3,2)}\right| \leqslant \exp \left(c \frac{\log M}{\log \log M}\right) .
$$

14. Next we consider the contribution of the $\varrho$ 's to $F_{M{ }_{3}}(s)$. This is obviously

$-\sum_{k \leqslant M} \frac{\mu(k) \log (M / k)}{\varphi(k)} \sum_{\chi \bmod k} \sum_{(\varrho)} L(s-\varrho, k, \bar{\chi}) \frac{1-e^{-\omega_{1} \varrho}}{\varrho(1+\varrho / \omega)^{\delta+1}} \stackrel{\text { def }}{=} F_{M ! 3}^{(3)}(s)$ and can obviously be written as

$$
\sum_{(n)} \frac{e_{n}^{(3,3)}}{n l^{s}}
$$

with

(14.2)

$$
e_{n}^{(3,3)}=-\sum_{\substack{k \leq M \\(k, n)=1}} \frac{\mu(k) \log (M / k)}{\varphi(k)} \sum_{x \bmod k}(x) \bar{\chi}(n, k) \sum_{\varrho(x)} n^{\varrho} \frac{1-e^{-\omega_{1} \varrho}}{\varrho(1+\varrho / \omega)^{\delta+1}} .
$$

This shows again that the series in (14.1) converges (absolutely) in a halfplane $\sigma>c$. This gives for $n=N$

$$
e_{N}^{(3,3)}=-\sum_{\substack{k \leqslant M \\(k, N)=1}} \frac{\mu(k) \log (M / k)}{\varphi(k)} \sum_{\chi \bmod k j} \bar{\chi}(N, k) \sum_{(\varrho(x))} N^{\varrho} \frac{1-e^{-\omega_{1} \varrho}}{\varrho(1+\varrho / \omega)^{\delta+1}} .
$$

Next we estimate trivially the contribution of $\varrho$ 's with

$$
|\gamma|>\omega M^{3 / 2 \delta} \text {. }
$$

This cannot exceed absolutely the quantity

$$
\begin{aligned}
4 M \log M \sum_{k \leqslant M} & \frac{1}{\varphi(k)} \sum_{x \bmod k}(x) \sum_{\gamma>\omega M^{3 / 2 \delta}} \frac{1}{\gamma\left(1+\gamma^{2} / \omega^{2}\right)^{(\delta+1) / 2}} \\
& <c M^{2} \log ^{2} M \sum_{\nu>\omega M^{3 / 2 \delta}} \frac{\log \nu}{\nu\left(1+\nu^{2} / \omega^{2}\right)^{(\delta+1) / 2}} \\
& <c M^{2} \log ^{2} M \cdot \omega^{\delta+1} \sum_{\nu>\omega M^{3 / 2 \delta}} \nu^{-\delta-2} \log \nu \\
& <c M^{2} \log ^{3} M \log \omega \cdot M^{-\frac{3}{2 \delta}(\delta+1)}<c \sqrt{M} \log ^{4} M
\end{aligned}
$$

using (6.3) and (6.1). Hence

$$
\begin{aligned}
& \text { (14.5) } \quad e_{N}^{(3,3)}=-\sum_{\substack{k \leqslant M I \\
(k, N)=1}} \frac{\mu(k) \log (M / k)}{\varphi(k)} \sum_{\chi \bmod (k)} \bar{\chi}(N, k) \times \\
& \times \sum_{|\gamma| \leqslant \omega M M^{3 / 2 \delta}} N^{e} \frac{1-e^{-\omega_{1} e}}{\varrho(1+\varrho / \omega)^{\delta+1}}+O\left(\sqrt{M} \log ^{4} M\right) .
\end{aligned}
$$

Now we have in a half-plane $\sigma>c$ the identity

$$
F_{M}(s)=F_{M 2}(s)+F_{M 4}(s)+F_{M 3}^{(1)}(s)+F_{M 3}^{(2)}(s)+F_{M 3}^{(3)}(s),
$$

and all these developable into Dirichlet series convergent here. Hence by the uniqueness theorem we have

$$
a_{N}=e_{N}^{(2)}+e_{N}^{(4)}+e_{N}^{(3,1)}+e_{N}^{(3,2)}+e_{N}^{(3,3)} .
$$

Collecting the corresponding expressions, we get

$$
\begin{aligned}
& \text { (14.6) } \sum_{p_{1}+p_{2}=N} \log p_{1} \log p_{2}=B_{1} N \prod_{\substack{p_{\mid} \mid N(N) \\
p_{>}}} \frac{p-1}{p-2}\left\{1+O\left(\frac{\delta}{\omega}\right)+O\left(\frac{1}{\log M}\right)\right\}-
\end{aligned}
$$

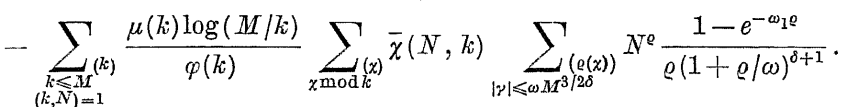

As to the left side we have evidently

$$
\left.\sum_{p_{1}+p_{2}=N} \log , p_{2}\right) \log p_{1} \log p_{2} \leqslant v_{2}(N) \log ^{2} N
$$

On the other hand we have

$$
\begin{aligned}
\sum_{p_{1}+p_{2}=N}\left(p_{1}, p_{2}\right) & \log p_{1} \log p_{2} \geqslant \sum_{\begin{array}{c}
p_{1}+p_{2}=N \\
\min p_{j} \geqslant N / \log N
\end{array}} \log p_{1} \log p_{2} \\
& \geqslant \log ^{2} N\left(1-8 \frac{\log \log N}{\log N}\right)\left\{v_{2}(N)-\sum_{\substack{p_{1}+p_{2}=N \\
\min p_{j}<N / \log ^{2} N}} 1\right\} \\
& \geqslant \nu_{2}(N) \log ^{2} N\left(1-9 \frac{\log \log M}{\log M}\right\}-c \frac{M}{\log M} .
\end{aligned}
$$

Hence

$$
\sum_{p_{1}+p_{2}=N} \log p_{1} \log p_{2}=v_{2}(N) \log ^{2} N\left\{1+O\left(\frac{\log \log M}{\log M}\right)\right\}+O\left(\frac{M}{\log M}\right)
$$


putting it into (14.6) we get

$$
\begin{aligned}
& \text { (14.7) } \quad v_{2}(N)=B_{1} \frac{N}{\log ^{2} N} \prod_{\substack{p \mid N \\
p>2}} \frac{p-1}{p-2}\left\{1+O\left(\frac{\delta}{\omega}\right)+O\left(\frac{\log \log M}{\log M}\right)\right\}-
\end{aligned}
$$

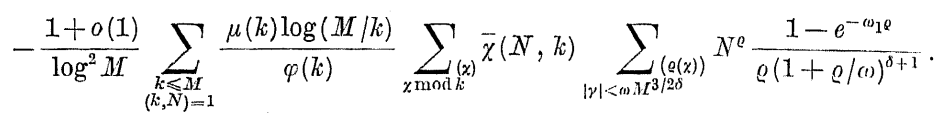

Until now $\delta, \omega$ and $\omega_{1}$ were restricted only by (6.1)-(6.2)-(6.3)-(6.4). Let $\varepsilon(x)>0$ and tending for $x \rightarrow \infty$ monotonically to 0 arbitrarily slowly but so that

$$
\lim _{x \rightarrow \infty} \varepsilon(x) \sqrt{\log x}=+\infty
$$

Then if we choose

$$
\omega=\log M, \quad \omega_{1}=0,99 \log N, \quad \delta=[\varepsilon(M) \log M]
$$

(14.7) takes the form

$$
\begin{aligned}
& y_{2}(N)= B_{1} \frac{N}{\log ^{2} N} \prod_{\substack{p \mid N \\
p_{>2}}} \frac{p-1}{p-2}\{1+O(\varepsilon(M))\}- \\
&- \frac{1+o(1)}{\log ^{2} M} \sum_{\substack{k \leqslant M \\
(k, N)=1}} \frac{\mu(k) \log (M / k)}{p(k)} \times \\
& \times \sum_{\chi \bmod k} \bar{\chi}(N) \\
& \bar{\chi}(N, k) \sum_{|\gamma| \leqslant \log M} \sum_{M \exp (3 / 2 \varepsilon(M))} \frac{N^{e}-N^{e / 100}}{\varrho(1+\varrho / \log M)^{[\varepsilon(M) \log M]+1}}
\end{aligned}
$$

which proves Theorem II.

\section{Appendix}

15. For the proof of Theorem I we start from the function for $\sigma>1$ ( $D$ positive even)

(15.1) $\quad H_{N}(s) \stackrel{\text { def }}{=}-\sum_{\substack{k \leqslant N+D) \\(k, D)=1}} \frac{\mu(k) \log ((N+D) / k)}{\varphi(k)} \sum_{x \bmod k}(x)=\frac{L^{\prime}}{\chi}(-D, k) \frac{L^{\prime}}{L}(s, k, \chi)$.
Substituting the Dirichlet series we get as previously

$$
\begin{aligned}
H_{N}(s)= & \sum_{\substack{n \leq N \\
(n, D)=1}} \frac{A(n) A(n+D)}{n^{s}}+ \\
& +\sum_{\substack{n \leq N \\
(n, D)>1}} \frac{\Lambda(n)}{n^{s}} \sum_{\substack{k, N+D) \\
(k, n)=(k, D)=1 \\
k \mid(n+D)}} \mu(k) \log \frac{N+D}{k}+\sum_{n>N} \frac{d_{n}}{n^{s}}
\end{aligned}
$$

Writing $H_{N}(s)$ in the form

$$
\begin{aligned}
& \text { (15.3) } \quad H_{N}(s)=-\sum_{\substack{k \leq N+D) \\
(k, D)=1}} \frac{\mu(k) \log ((N+D) / k)}{\varphi(k)} \times \\
& \times \sum_{k^{*} \mid k} \sum_{\left(k^{*}\right)} \sum_{z^{*} \bmod \left(k^{*}\right)}^{*} \bar{x}(-D, k) \frac{L^{\prime}}{L}\left(s, k^{*}, \chi^{*}\right)-\sum_{\substack{k \in N+D \\
(k, D)=1}} \frac{\mu(k) \log ((N+D) / k)}{\varphi(k)} \times \\
& \times \sum_{k^{*} \mid k} \sum_{\left(k^{*}\right)} \sum_{\chi^{*} \bmod k^{*}}^{*} \bar{\chi}^{*}\left(-D, k^{*}\right) \sum_{p \mid \frac{k}{k^{*}}} \frac{\chi^{*}\left(p, k^{*}\right) \log p}{p^{s}-\chi^{*}\left(p, k^{*}\right)} \stackrel{\text { def }}{=} H_{N_{1}}(s)+H_{N_{2}}(s)
\end{aligned}
$$

we start from the integral

$$
\text { (15.4) } I=I_{1}+I_{2} \text {, }
$$

where, with $\alpha>1$,

$$
I_{j}=\frac{1}{2 \pi i} \int_{(\alpha)} \frac{N^{s}\left(1-e^{-\omega_{3} s}\right)}{s\left(1+s / \omega_{2}\right)^{\delta_{1}+1}} H_{N j}(s) d s, \quad j=1,2,
$$

and $\delta_{1}, \omega_{2}, \omega_{3}$ are restricted at present only by

$$
3 \leqslant \omega_{2} \leqslant \log N,
$$

$$
\delta_{1}>3 \log \log N \quad \text { and integer, }
$$

$$
\frac{\delta_{1}}{\omega_{2}} \rightarrow 0
$$

$$
3 \log \log N \leqslant \omega_{3} \leqslant 0,99 \log N \text {, }
$$

$$
2 \leqslant D \leqslant \frac{N}{\log ^{10} N}
$$

and they will be determined exactly later.

16. Using the Dirichlet series representation of $H_{N}(s)$ in (15.2) and also Lenma I, we get (with the notation (6.8) but with $\delta_{1}$ instead of $\delta$ 
and similarly with the $\omega$ 's)

(16.1) $\quad I=\sum_{\substack{n \leq N \\(n, D)=1}} \Lambda(n) \Lambda(n+D) b(N, n)+$

$$
+\sum_{\substack{n \leqslant N \\(n, D)>1}} A(n) b(N, n) \sum_{\substack{k \leqslant N+(k) \\(k, n)=(k, D)=1 \\ k \mid(n+D)}} \mu(k) \log \frac{N+D}{k} .
$$

The second sum in (16.1) is absolutely, as is easy to see,

$$
\leqslant c \log ^{z}(N+D) \max _{1 \leqslant l \leqslant N+D} \tau(l) \sum_{p \mid D}(p) \sum_{p^{\alpha} \leqslant N} 1<c N^{1 / 10} .
$$

In the first sum of (16.1) Schnirelman's theorem implies, together with (15.8)

$$
\sum_{\substack{\delta_{1} \\ N \exp (-2 \\(n, D)=1}} \Lambda(n) \Lambda(n+D) b(N, n)<c N \frac{\delta_{1}}{\omega_{2}} \prod_{\substack{p>2 \\ p>D}} \frac{p-1}{p-2}
$$

and evidently from (15.9) and (15.10)

$$
\sum_{n \leqslant N e^{--\omega_{3}}} \Lambda(n) \Lambda(n+D) b(N, n)<c \log ^{2} N \cdot N e^{-\omega_{3}}<c \frac{N}{\log N} .
$$

Hence we got from (16.1)

$I=\sum_{\substack{\left.p_{1}=p_{2}+D \\ p_{1}, p_{2}\right)}} \log p_{1} \log p_{2} \cdot b\left(N, p_{2}\right)+O(N)\left\{\frac{\delta_{1}}{\omega_{2}}+\frac{1}{\log N}\right\} \prod_{\substack{p \mid D \\ p \leqslant p_{2}}} \frac{p-1}{p-2}$

and on using (15.7) and (6.9)(c)

(16.2) $I=\sum_{\substack{p_{2} \leqslant N \\ p_{1}=p_{2}+D}} \log p_{1} \log p_{2}+O(N) \prod_{\substack{p \mid D \\ p>2}} \frac{p-1}{p-2}\left(\frac{\delta_{1}}{\omega_{2}}+\frac{1}{\log N}\right)$.

17. Next we investigate $I_{2}$ in (15.4). This gives as in 10

$$
\begin{aligned}
& I_{2}=-\sum_{\substack{k \leqslant N+D \\
(k, D)=1}} \frac{\mu(k) \log ((N+D) / k)}{\varphi(k)} \sum_{k^{*} \mid k} \sum_{k *} \sum_{\chi^{*} \bmod \left(k^{*}\right)} \sum_{(k)}^{*} \overline{\chi^{*}}\left(-D, k^{*}\right) \times \\
& \times \sum_{p \mid \frac{k}{k^{*}}} \log p \sum_{\lambda=1}^{\infty}\left({ }^{*} \chi^{*}\left(p^{\lambda}, k^{*}\right) b\left(N, p^{\lambda}\right)=\sum_{\substack{k \leqslant N+D \\
(k, D)=1}} \frac{\mu(k) \log ((N+D) / k)}{\varphi(k)} \times\right. \\
& \times \sum_{k^{*} \mid k} \sum_{\left(k^{*}\right)} \log p \sum_{p \mid \frac{k}{k^{*}}}(p) b\left(N, p^{\lambda}\right)\left\{\sum_{x^{*} \leqslant N+D^{*}}\left(\sum_{\bmod k^{*}}^{*} \chi^{*}\left(p^{\lambda}(-D)^{-1}, k^{*}\right)\right\} .\right.
\end{aligned}
$$

Using Lemma IV and (6.9)(b), we get

$$
\left|I_{2}\right| \leqslant \log ^{2}(N+D) \sum_{\substack{k \leqslant N \\(k, D)=1}} \frac{|\mu \cdot(k)|}{\varphi(k)} \sum_{k^{*} \mid k} \sum_{\left(k^{*}\right)} \sum_{p \mid \frac{k}{k^{*}}} \sum_{p^{2} \leqslant N+D}\left(p^{2}+D, k^{*}\right) .
$$

The next steps are quite analogous to those in 10 (after (10.3)) and result

$$
\left|I_{2}\right|<\exp \left(c \frac{\log N}{\log \log N}\right) .
$$

Next we consider $I_{1}$. Shifting the line of integration to the line Res $=-0,99$ let the contribution of the residua resp. of the integral be $I_{3}$ resp. $I_{4}$. For $I_{4}$ we get from (15.1) and (15.5)

$$
\begin{aligned}
& \text { (17.2) }\left|I_{4}\right| \\
& \leqslant c\left(\frac{e^{\omega_{3}}}{N}\right)^{0,99} \log (N+D) \sum_{k \leqslant N+D} \frac{1}{(k)} \frac{\sum_{\varphi}(k)}{z \sum_{\bmod }(k)} \int_{0}^{\infty} \frac{\log \{(N+D)(2+v)\} d v}{(1+v)\left\{\left(1-1 / \omega_{2}\right)^{2}+v^{2} / \omega_{2}^{2}\right\}^{\left(\delta_{1}+1\right) / 2}} \\
& <c N \log N\left\{\log ^{2} N+\frac{1}{N} \int_{N}^{\infty} \frac{\log N v}{v^{\delta_{1}+1}} \omega_{2}^{\delta_{1}+1} d v\right\}\left(\frac{e^{\omega_{3}}}{N}\right)^{0,99} \\
& <c N \log ^{3} N\left(\frac{e^{\omega_{3}}}{N}\right)^{0,99}=O\left(\frac{N}{\log N}\right)
\end{aligned}
$$

owing to (15.9) and (15.6). Collecting (16.2), (17.1) and (17.2), we get

$$
\text { (17.3) } \sum_{p_{2} \leqslant N, p_{1}=p_{2}+D} \log p_{1} \log p_{2}=I_{3}+O(N)\left\{\frac{\delta_{1}}{\omega_{2}}+\frac{1}{\log N}\right\} \prod_{\substack{p \mid D \\ p>2}} \frac{p-1}{p-2} \text {. }
$$

18. Finally we consider the residua. The contribution of the poles at $s=1$ is

$$
\text { (18.1) } \begin{aligned}
N \frac{1-e^{-\omega_{3}}}{\left(1+1 / \omega_{2}\right)^{\delta_{1}+1}} & \sum_{\substack{k \leqslant N+D \\
(k, D)=1}} \frac{\mu(k) \log ((N+D) / k)}{\varphi(k)} \\
& =B_{1} N \prod_{\substack{p>2 \\
p>D}} \frac{p-1}{p-2}\left\{1+O\left(\frac{\delta_{1}}{\omega_{2}}\right)+O\left(\frac{1}{\log N}\right)\right\}
\end{aligned}
$$

owing to Lemma III, (15.8) and (15.9).

The contribution of residua at $s=0$ is

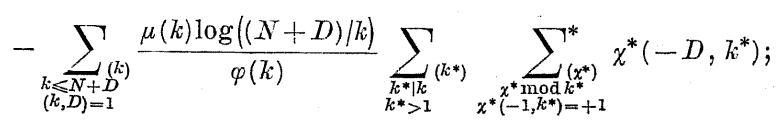


the reasoning in 13 gives mutatis mutandis for the absolute value of this expression the upper bound

\section{References}

$$
c \log ^{2} N \sum_{k \leqslant N+D} \frac{|\mu(k)|}{\varphi(k)} \sum_{k^{*} \mid k}\left\{\left(D+1, k^{*}\right)+\left(D-1, k^{*}\right)\right\}
$$

i.e.

$$
\exp \left(c \frac{\log N}{\log \log N}\right)
$$

using also (15.10). Finally the contribution of the nontrivial zeros is

$$
-\sum_{\substack{k \leqslant N+D \\(k, D)=1}} \frac{\mu(k) \log ((N+k) / D)}{\varphi(k)} \sum_{x \bmod k} \bar{\chi}(-D, k) \sum_{(\varrho(x))} \frac{N^{\varrho}\left(1-e^{-\omega_{3} \varrho}\right)}{\varrho\left(1+\varrho / \omega_{2}\right)^{\delta_{1}+1}}
$$

The reasoning in 14 gives mutatis mutandis that the contribution of $\varrho$ 's with

is $O\left(N^{3 / 4}\right)$.

$$
|\gamma|>\omega_{2}(N+D)^{3 / 2 \delta_{1}}
$$

19. Let $\varepsilon(x)$ be positive tending to 0 for $x \rightarrow+\infty$ monotonically arbitrarily slowly but so that

$$
\varepsilon(x) \sqrt{\log x} \rightarrow+\infty
$$

Then choosing

$$
\begin{gathered}
\omega_{3}=0,99 \log N, \\
\delta_{1}=[\varepsilon(N) \log N], \\
\omega_{2}=\log N, \\
N>\max \left(c, D \log ^{10} D\right)
\end{gathered}
$$

the requirements (15.6)-(15.10) are fulfilled and

$$
\begin{aligned}
& \sum_{\substack{p_{2} \leqslant N \\
p_{1}=p_{2}+D}} \log p_{1} \log p_{2} \\
& =\{1+O(\varepsilon(N))\} B_{1} N \prod_{\substack{p \mid D \\
p>2}} \frac{p-1}{p-2}-\sum_{\substack{k \leqslant N+D) \\
(k, D)=1}} \frac{\mu(k) \log ((N+D) / k)}{\varphi(k)} \times \\
& \quad \times \sum_{x \bmod k} \bar{\chi}(-D, k) \sum_{|\gamma| \leqslant \log N \exp (3 / 2 \varepsilon(N))} \frac{N^{e}-N^{e / 100}}{\varrho(1+\varrho / \log N)^{[\varepsilon(N) \log N]+1}} .
\end{aligned}
$$

Then the proof can be completed as in Theorem II.

[1] G. H. Hard $y$ and J. E. Littlewood, Some problems of partitio numerorum III. On the expression of a number as a sum of primes, Acta Math. 44 (1922), pp. 1-70. [2] K. Prachar, Primzahlverteilung, Berlin 1957.

[3] P. Turán, On the twin prime problem I, Publ. Math. Inst. Hung. Acad. Sei. Ser. 4. 9 (3) (1964), pp. 247-261.

[4] П. Т у ран, О некоторых пеоретико-функииональных методах решета 6 mеории чисел, Докл. Акад. Наук СССР 171 (1966), pp. 1289-1292. For a some more complete English translation under the title "Some functiontheoretic sieve methods in the theory of numbers" where also some misprints are corrected see Soviet Math. Dokłady $7(6)$ (1966), pp. 1661-1664.

Reģu par la Rédaction le 3. 10. 1966 . 